\title{
Organizaciones en red Factores críticos de diseño
}

\author{
Gladis Cecilia Villegas Arias*
}

\section{Resumen}

En este trabajo se presentan los resultados de una investigación orientada a reconocer los factores críticos de éxito al diseño de cuatro redes productivas, operantes en el sector agrícola en Colombia: producción y comercialización de café, comercialización de banano, procesamiento de derivados lácteos y cultivo de flores.

Las redes incluidas en el estudio se sometieron a observación durante el periodo de julio de 2001 a julio de 2004; los datos recolectados se procesaron usando la metodología llamada teoría fundamentada (o inductiva); asimismo, se utilizó la codificación axial para priorizar las características comunes a las estructuras observadas, sus variables de diseño organizativo y las interacciones entre unas y otras; finalmente, con las temáticas identificadas se construyó un modelo de factores críticos de diseño para redes productivas.

Palabras clave: redes, teoría de la organización, factores críticos de éxito, teoría fundamentada (o inductiva)

Fecha de recepción: 12/02/2007

Fecha de aceptación: 06/12/2007

\section{Company Networks. Critical Design Factors}

\begin{abstract}
This paper presents the results of observing four (4) networks that do business in the agriculture sector in Colombia: Growers of coffee, bananas, flowers and producers of dairy products. The research was designed following the Grounded Theory method. Particularly, Axial Coding was applied to relate theoretical design variables to observed network behaviors with the purpose of identifying critical design factors for the organizations under study. A model relating the findings is included.
\end{abstract}

Key words: Networks, Organizational Design, Critical Success Factors, Agriculture Sector, Grounded Theory.

\footnotetext{
*Profesora-investigadora en la Universidad de Medellín, Antioquia, Colombia.

Correo electrónico: gcvillegas@udem.edu.co
} 
Gladis Cecilia Villegas Arias

\section{Introducción}

T as organizaciones productivas difieren de las sociales en que las primeras son

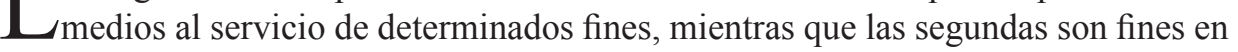
sí mismas. El diseño de las organizaciones productivas debe alinearse a la naturaleza de la estrategia (Miller, 1987; Bart, 1986; Egelhoof, 1982; Miller, 1986; Chandler, 1988; White, 1986, Engdahl y otros, 2000), la misma que se ajusta a los cambios en el entorno social y de negocios (Barney, 1986; Grant, 1996; Irenland y otros, 1987; Miller, 1987; Porter, 1997; Prescott, 1986); en otras palabras, las productivas son organizaciones de diseño dinámico y previo a la vinculación de sus actores.

Las distintas condiciones socioeconómicas dieron origen a ciertas estructuras típicas; por ejemplo, la producción artesanal del medioevo originó el mutualismo gremial (Huberman, 1996; Rule, 1990), mientras que el predominio de la producción en masa impulsó la aparición de la burocracia jerárquica basada en el control, la especialización del trabajo y la estandarización de procesos (Fayol y Taylor, 1969).

La sociedad ha cambiado de nuevo; en lo cultural, el predominio del individuo ha modificado los patrones de demanda por productos personalizados, que se ajusten a las preferencias y singularidades del consumidor. La producción masiva ha caído en desuso e impuesto la urgencia de una producción flexible y ágil.

Por otro lado, los costos de gobierno de una organización crecen con la complejidad de la misma (Williamson, 1996). Si se mide la complejidad, como número de partes y relaciones entre ellas, podemos entender que el crecimiento organizacional tiene una relación directa con los costos de gobierno. Estructuras más grandes reportan menos ganancias porque su funcionamiento es más oneroso.

Una forma organizativa moderna, basada en la cooperación entre empresas, se viene imponiendo en el mundo. Esta forma organizativa denominada "organización en red" consiste en la alianza a largo plazo de clientes y proveedores para la ejecución de tareas que, desde la estructura integrada, acostumbraban a estar bajo el control de una misma organización.

Pocos estudios han comparado el funcionamiento de redes productivas para teorizar sobre sus fundamentos de diseño. Este trabajo revisó la literatura relevante; seleccionó variables propuestas como importantes y las utilizó como referente para la observación de cuatro organizaciones actuantes en el sector agrario en Colombia; reconoció elementos comunes a la operación de las entidades observadas; perfeccionó la lista de variables inicial para, finalmente y con base en la evaluación de interac- 
Organizaciones en red

Factores críticos de diseño

ciones entre los datos obtenidos, proponer un conjunto de elementos imprescindibles a la existencia de ese tipo de estructuras.

La pregunta investigativa que este trabajo respondió es: ¿cómo se relacionan las características de una organización en red con las variables propias del diseño organizativo? Los objetivos específicos para la investigación fueron:

- Identificar las características comunes a la identidad de las estructuras productivas en red.

- Reconocer las variables de diseño organizativo más influyentes cuando de diseñar ese tipo de estructuras se trata.

- Relacionar las características observadas a las variables de diseño con el fin de descubrir los factores críticos de diseño de redes.

\section{Fundamentación teórica}

\subsection{Definición}

Al nivel más general, una red productiva es un sistema de empresas y entidades relacionadas entre sí por un modelo de negocio que les es común (Podolny y Page, 1998). Las redes observadas en este estudio, por ejemplo, agrupan a empresarios que tienen producción local y comercialización nacional e internacional. Las empresas participantes se acogen al modelo de negocio colectivamente formulado y ejecutado, lo que es un requisito de asociación.

\subsection{Antecedentes históricos}

La revolución industrial convocó agrupamientos humanos no naturales, sometidos a unas disciplinas y comportamientos predeterminados, que eran necesarios para lograr resultados predefinidos (Toffler, 1993; 1999).

$\mathrm{Al}$ frente de las masas humanas involucradas en las nuevas formas de producción se colocaron directores dotados de autoridad suficiente para ejercer control al más tradicional estilo militar (Morgan, 2006).

Las milicias empresariales, provenientes de este ejercicio organizativo, resultaron eficientes para atender mercados demandantes de gran volumen, tecnologías de producción a gran escala y mano de obra pobremente capacitada que se emplease como apéndice de las máquinas (Daft y Lengel, 1984). 
El lado oscuro de estas estructuras se evidenció en la división, concentración y excesiva especialización del trabajo en parcelas internas cuyos directores construyeron verdaderos feudos de lealtad incondicional a su nombre. Cada grupúsculo exhibía una fuerte cohesión interna a la vez que una pobre o casi nula interacción con otros grupos (Mintzberg, 1982).

En el nivel económico, el fraccionamiento de la acción en múltiples e incomunicados esfuerzos elevaba los costos de operación y daba al traste con la efectividad; convirtiendo a la organización en un imperfecto instrumento para la operación empresarial (Majchrzak y Wang, 1996).

Cambios en los patrones de consumo y en los procesos productivos acabaron por inutilizar al estándar burocrático de organización. La producción en masa con su inagotable oferta de artículos idénticos tuvo que dar paso a una producción personalizada de artículos "hechos a la medida" a costo razonable (Toffler, 1993).

Al gigantismo organizacional tuvieron que oponerse formas livianas de respuesta rápida y procesos flexibles. Las teorías administrativas asociadas con adelgazamiento institucional siguen incluyendo palabras como: outsourcing (tercerización de procesos), downsizing (eliminación de unidades y reducción de planta de personal) y rightsizing (concentración en actividades que se ejecutan magistralmente) (Barreto, 1995; Prida y Gutiérrez, 1996; Harrigan, 1985; Venkatesan, 1992; Walker y Weber, 1987).

Sin embargo, ¿cómo garantizar que la nueva forma de especialización por empresas produzca resultados coherentes y de calidad?, ¿cómo hacerse parte de un desenlace sobre cuyos antecedentes no se tiene total control? Hacerse responsable por acciones ajenas parece un acto suicida. Las reflexiones inducidas por este tipo de preguntas prepararon el camino para la llegada de las redes como modelos asociativos modernos de producción.

Si ostentar la propiedad sobre múltiples etapas del proceso encarece los costos administrativos y de operación, la solución apunta a concentrarse en aquellas etapas en las que pueda demostrarse una superioridad evidente frente al desempeño de los competidores mientras que, a la vez, se fortalezcan las alianzas entre empresas.

En síntesis, producir en red demanda una ejecución efectiva, rentable y de calidad de los procesos elegidos y gran habilidad para manejar las relaciones de negocios con otras empresas. Ello puede lograrse mediante esquemas de cooperación tales como: intercambio de información crítica para la toma de decisiones, coordinación de actividades conjuntas, planeación colectiva, comunicación oportuna de eventualidades 
Organizaciones en red

Factores críticos de diseño

imprevistas, control a la ejecución de planes, negociación de apoyo y condiciones especiales con el gobierno y con otros actores económicos, entre otras.

La operación en red plantea nuevos retos a la gerencia: retos de sincronización de procesos, estandarización de medidas y lenguajes, negociación de acuerdos gana-gana, diseño acordado de sistemas de evaluación de desempeño, definición de planes de contingencia y adopción de sistemas de información interorganizacionales, entre otros.

El diseño de organizaciones es una metodología para configurar sistemas sociales. La tarea consiste en definir estructuras, sistemas gerenciales y redes de relaciones. A continuación se revisará la teoría de diseño con la intención de encontrar las variables más relevantes al caso de redes productivas.

\subsection{Variables clave del diseño de redes}

Se propone un listado de condiciones que serían propias al funcionamiento óptimo de una red. Este listado derivó de una revisión extensa de literatura en temas de diseño de organizaciones, comportamiento organizacional y estructuras en red. El listado de condiciones propuesto se validó con la observación y seguimiento, durante cuatro años, al funcionamiento de cuatro redes productivas que operan en el sector agrario en Colombia.

La teoría gerencial propone a la organización productiva como un fenómeno social, tecnológico y económico. En esta sección se sugieren y describen variables clave de diseño organizativo aplicables a la estructura en red en cada uno de los terrenos anteriores.

\section{A. Variables en el nivel tecnológico}

A.1. Estrategia de producción. La estrategia de producción se deriva de la estrategia corporativa y específica (Hayes y Wheelwright, 1984).

- Objetivos de producción a la luz de los objetivos de la red

- Cursos de acción que conlleven cumplimiento de esos objetivos

- Recursos necesarios para la ejecución de los planes

En una red, la estrategia de producción debe desagregarse en el nivel de cada empresa y controlar la ejecución para garantizar el cumplimiento armónico.

A.2. Programación de operaciones. Programar operaciones es agendar recursos necesarios para la operación respetando el diseño de procesos y actividades y satisfaciendo las restricciones existentes (Sipper y Bulfin, 1997). 
En una organización en red, la programación de operaciones tiene gran relación con la sincronización y con la gestión de intercambios (enlaces) entre empresas.

A.3. Gestión de inventarios. Conjunto de decisiones enfocadas a garantizar que los insumos o productos utilizados en una organización estén disponibles cuando se les necesite (Ballou, 2004).

A.4. Gestión logística. Conjunto de decisiones relativas a la gestión de inventarios, transporte e instalaciones para el cumplimiento de los niveles de servicio al cliente decididos a nivel estratégico (Ballou, 2004).

A.5. Sistemas de información y comunicaciones interorganizacionales. Infraestructura para el procesamiento de datos y transmisión de mensajes entre empresas participantes en el arreglo en red.

\section{B. Variables en el nivel económico}

B.1. Planeación. Selección de misiones, objetivos y cursos de acción necesarios para alcanzarlos (Weihrich y Koontz, 2004: 35).

B.2. Diseño organizativo. Establecimiento de una estructura intencionada de roles, funciones y relaciones que enmarca el accionar de los participantes (Weihrich y Koontz, 2004: 35).

B.3. Vinculación de participantes. Función a cargo de llenar y mantener ocupadas las posiciones definidas por el diseño organizativo.

B.4. Competitividad. "La competitividad es la habilidad para lograr que los clientes prefieran, en forma sostenible, el producto o servicio frente a las alternativas de la competencia" (Gaynor, 1999: 143).

B.5. Gestión de demanda vs. suministro. Sistema de decisiones enfocado a garantizar que las determinaciones de producción se informen de las condiciones de la demanda.

B.6. Sistema contable. "Sistema de información basado en el registro, clasificación, medición y resumen de cifras significativas que, expresadas en términos monetarios, muestran el estado de las operaciones realizadas por una organización" (Grupo Santander, "Glosario financiero", www.bves.com.sv/glosario/g_c.htm, 2 de febrero de 2007). 
Organizaciones en red

Factores críticos de diseño

B.7. Presupuesto. Expresión numérica de ingresos y egresos esperados para un periodo de tiempo y relacionados con ejecución de programas, proyectos o actividades.

B.8. Gestión de proyectos. Decisiones dirigidas a garantizar que los proyectos emprendidos alcancen los objetivos esperados en el tiempo y a los costos acordados (Meredith y Mantel, 2007).

B.9. Finanzas. "Rama de la administración que se preocupa de la obtención y determinación de los flujos de fondos requeridos y de distribuir y administrar esos fondos en los activos, plazos y fuentes de financiamiento con el objetivo de maximizar el valor económico de la organización" (BusinessCol.com; "glosarios", www.businesscol.com/glosarios/contable/glorasio_contable_f.html, 20 de diciembre de 2007).

\section{Variables en el nivel social}

C.1. Identidad. "Conjunto de rasgos propios de un individuo o de una colectividad que los caracteriza frente a los demás” (Diccionario de la Lengua Española, 2001).

C.2. Comunicación. Transmisión efectiva de mensajes, utilizando un sistema de signos y reglas, que puede ser entendido por emisor y receptor (Diccionario de la Lengua Española, 2001).

C.3. Gestión. Acción y efecto de decidir sobre la aplicación de recursos a la obtención de más recursos.

C.4. Relaciones. Conexiones, correspondencias, tratos y comunicaciones de una entidad con otra (Diccionario de la Lengua Española, 2001).

C.5. Liderazgo. Ejercicio del reconocimiento que un ente tiene entre los de su clase para influir en su comportamiento (Weihrich y Koontz, 2004).

C.6. Negociación. Acción y efecto de llevar a cabo tratos dirigidos a la realización de un acuerdo (Diccionario de la Lengua Española, 2001).

C.7. Confianza. Convicción que se tiene de que una persona o cosa posee las cualidades y producirá los comportamientos o resultados esperados (Kramer y Tyler, 1996; Luhmann, 1996).

C.8. Compromiso. Obligación, voluntariamente contraída, de desempeñarse al mayor nivel posible de las propias competencias. 
Gladis Cecilia Villegas Arias

\subsubsection{Relaciones entre variables de diseño}

Las variables de diseño no son independientes entre sí. La estimación del grado de interacción, propuesta a continuación, está basada en la aplicación de la teoría gerencial al caso de redes, en la observación del funcionamiento de cuatro de dichas estructuras en Colombia y en la experiencia laboral, docente e investigativa de la autora.

\section{A. Variables en el nivel tecnológico}

A.1. La estrategia de producción planea la operación de la red en sus objetivos, acciones y recursos cuidando que la oferta de bienes y servicios esté en consonancia con la demanda de los mismos. Al igual que la estrategia corporativa, de la cual depende, tiene en la estructura a su medio de acción y principal limitante; asimismo, su implementación está a cargo del sistema de gestión. Algunas de las actividades más significativas a la ejecución de esta estrategia son: programación de operaciones, gestión de inventarios y gestión logística.

A.2. La programación de operaciones es la actividad de gestión dedicada a agendar la ejecución del trabajo. Su nivel de efectividad impacta de manera significativa a los costos de operación, los mismos que son registrados por el sistema contable. Consulta a los niveles de demanda real y estimada, se aplica a la gestión logística y, cuando así lo hace, determina los niveles de inventario requeridos. Como cualquier otra tarea, demanda una apropiación presupuestal adecuada.

A.3. La gestión de inventarios es la actividad de la gestión logística a cargo de conseguir los inventarios necesarios para satisfacer la demanda. Los costos de sus decisiones quedan registrados en el sistema contable.

A.4. La gestión logística tiene por objetivo la satisfacción de la demanda mediante el movimiento de inventario a los lugares, momentos, precios, actores y en las condiciones requeridas. Tanto los procesos comunicativos como sus tecnologías asociadas son herramientas fundamentales al éxito de esta gestión. En sí misma, ella es un elemento decisivo a la competitividad de la red.

A.5. Los sistemas interorganizacionales de información y comunicación son resultado del proceso de diseño organizacional. Estos sistemas son herramientas imprescindibles a la gestión de redes debido a que, entre otras cosas, permiten identificar las tendencias de la demanda y con ese conocimiento decidir acerca de la producción futura. Esta plataforma apoya los procesos de información y comunicación a través de la red y configuran el tejido invisible de la estructura. 
Organizaciones en red

Factores críticos de diseño

\section{B. Variables en el nivel económico}

B.1. La planeación apunta a lograr que la organización sea competitiva, objetivo que demanda de un diseño de la estructura adecuado a la ejecución de las acciones que los planes requieren.

B.2. El diseño organizativo se relaciona, por un lado, con los sistemas de comunicación debido a que éste los contiene; por otro, con la gestión, pues diseñar organizaciones es una de sus funciones clásicas.

B.3. La multiplicidad de intereses que se dan cita en un arreglo productivo en red es tal, que la operación conjunta sería imposible sin la confianza mutua entre actores; de tal modo, cuando se vinculan participantes, sean individuos o empresas, se están construyendo las bases de lo que será la identidad del sistema —una identidad construida en bases de confianza mutua y comunidad de objetivos.

B.4. La competitividad de una red depende de la calidad de su gestión

B.5. Una de las tareas de la gestión es alinear la oferta con la demanda.

B.6. Tanto el sistema contable como el sistema financiero monitorean la adquisición, uso e inversión del recurso capital. Un ejemplo de ellos es el registro de los recursos invertidos en la ejecución de proyectos.

B.7. Todo proyecto necesita de recursos. La situación financiera de la red afecta a la capacidad de destinación presupuestal y, por tanto, restringe el número y tipo de proyectos que se pueden emprender.

B.8. Gestión de proyectos y comunicación interactúan en que la comunicación es crucial a la gestión exitosa de proyectos; de igual manera, la relación entre gestión de proyectos y gestión es que la primera es una aplicación de la segunda.

\section{Variables en el nivel social}

C.1. Los procesos de identidad se construyen a partir de las relaciones interpersonales, se suceden en el universo del lenguaje y las comunicaciones, son más fuertes cuanto mayor sea la confianza entre los actores. El estilo de liderazgo es uno de los generadores de identidad en cuanto tiende a ser generalmente imitado. Asimismo, la identidad puede evidenciarse en comportamientos tales como los estilos adoptados para la negociación. 
C.2. La fortaleza de las relaciones en las sociedades humanas depende de la calidad de sus comunicaciones. Es la comunicación la que perfecciona la confianza entre los humanos y les induce al compromiso legítimo. La comunicación efectiva es un recurso imprescindible para la gestión especialmente porque sin ella no puede hablarse de liderazgo legítimo y porque es un ingrediente diferenciador en los procesos de negociación

C.4. El ejercicio de relacionarse con el otro es el escenario natural al desarrollo de la confianza, el compromiso y el liderazgo; adicionalmente, las formas de relacionarse sientan el precedente de una historia que incide en asuntos tales como los estilos de negociación que se adopten por los involucrados.

C.5. Líder es quien se compromete con un propósito; cabe aclarar que no se reconoce como líder a quien no inspira confianza.

C.6. Sin compromiso es imposible hacer efectivos los acuerdos negociados.

C.7. Creer en el otro equivale a percibirle como a un ser que se compromete. 
Organizaciones en red

Factores críticos de diseño

Tabla 1

Correlaciones entre variables clave de diseño

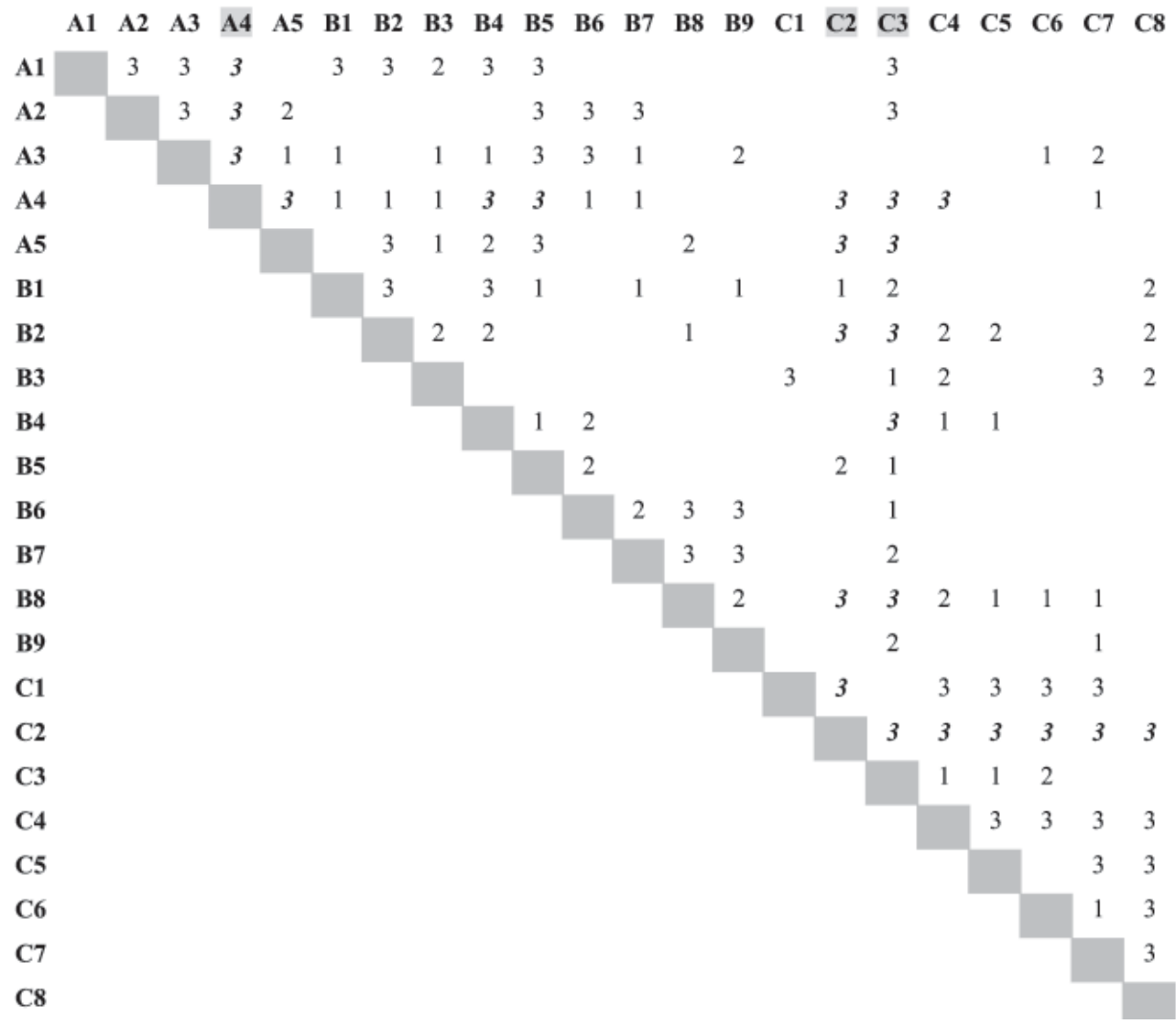

La tabla 1 ofrece una valoración cualitativa de la intensidad de la interacción entre variables típicas de diseño. La nomenclatura utilizada indica una relación fuerte cuando se asigna el valor de 3; una relación media para un valor de 2 y una relación débil para un valor de 1 .

El análisis nos revela que las variables más interrelacionadas con otras son: Gestión (C3) con 17 (diecisiete conexiones); Gestión logística (A4) con 15 (quince) conexiones y Comunicación (C2) con 13 (trece) conexiones. Por otro lado, las variables con mayor intensidad en sus relaciones son: Comunicación (C2) con 11 (once) conexiones fuertes de 13 (trece); Gestión logística (A4) con 9 conexiones fuertes de 15 (quince) y Gestión (C3) con nueve conexiones fuertes de 17. Considerando la intensidad y cantidad de conexiones, las variables se clasificarían en orden de centralidad (impacto), así: Gestión, Comunicación y Gestión logística. 
Si admitimos que todo diseño organizativo tiene dos dimensiones, una encargada de la dirección y otra encargada de la operación (Blanchard, Ken, 1996), los resultados anteriores reconocen la trascendencia nuclear de estos elementos y de la comunicación como mecanismo articulador por excelencia entre ambos.

\section{Metodología}

El método de investigación empleado fue la teoría fundamentada (también llamada inductiva). Se partió del marco teórico del diseño de organizaciones y su aplicación al diseño de redes para orientar la observación del funcionamiento de cuatro (4) redes productivas que operan en el sector agrícola colombiano: exportadores de café, exportadores de banano, exportadores de flores y procesadores de leche de vaca. Las redes incluidas en el estudio fueron elegidas por ser las más representativas de la economía legal colombiana. Tres de ellas exportan sus productos al mercado internacional.

Las redes están constituidas por empresas agrícolas y de alimentos de diversos tamaños que se asocian para hacerse más fuertes en el mercadeo de sus productos, en la negociación con el gobierno, en el establecimiento de sistemas de calidad y en la inversión en actividades de investigación y desarrollo.

Se reunió información de múltiples fuentes utilizando distintas técnicas que incluyeron: revisión de bibliografía; revisión de noticias, documentos y publicaciones internas; entrevistas a actores clave; observación participativa a la ejecución de actividades de rutina; cuestionarios semiabiertos (aplicados a productores de café y cultivadores de banano) y revisión de aspectos específicos por parte de estudiantes en trabajo de campo. La recolección de datos se llevó a cabo en el periodo de julio de 2001 a julio de 2004.

Los informantes fueron seleccionados con el criterio de involucrar la mayor variedad posible de visiones e intereses al interior de cada organización. Igual criterio se aplicó a la selección de fuentes (publicaciones en medios externos independientes, informes estatales, periódicos internos y externos y documentos históricos) y a la utilización de técnicas (observación, revisión documental y entrevistas).

Se utilizó la técnica de análisis cualitativa denominada codificación axial para priorizar las características comunes a las estructuras observadas, sus variables de diseño organizativo y las interacciones entre unas y otras. El resultado de este ejercicio evidenció las temáticas comunes y esenciales a la supervivencia de las organizaciones examinadas. Finalmente, los resultados se incluyeron en un modelo de factores críticos al diseño de redes productivas. 
Organizaciones en red

Factores críticos de diseño

Las cuatro (4) redes observadas participan del sector agrícola colombiano y son representativas del fenómeno en el país, mientras pueden no serlo de éste en otros lugares, lo que afecta la generalización de resultados a otros contextos.

\section{Resultados}

\subsection{Condiciones ideales}

La información recolectada permitió reconocer las siguientes características comunes a la operación de las redes observadas:

1. Existencia de una estrategia corporativa pensada para la red y desagregada a nivel de cada empresa participante.

2. Modelo de negocios colectivo que define mercados, líneas de productos y canales de distribución para la red vista como un todo.

3. Operación de procesos sincronizados con base en predicciones de demanda compartidas y programación conjunta.

4. Sistemas de control de calidad acordados y funcionando adecuadamente.

5. Negociación de precios basada en márgenes de ganancia.

6. Sistema encargado de la gestión de la red, que cuenta con mecanismos de representación de todos los actores.

7. Definición, comunicación y aplicación de perfiles para la selección de candidatos a participar en la red.

8. Diseño dinámico de red, con mecanismos de alineación a la estrategia en uso y suficientemente concertado para servir de base a los acuerdos de negocio.

9. Red logística adecuadamente modelada y con mecanismos de retroalimentación que permiten la optimización dinámica.

10. Sistemas de costeo compartidos y de amplio acceso.

11. Sistemas y protocolos de comunicación bien diseñados y efectivamente utilizados por todos los participantes. 
12. Existencia de programas de mejoramiento continuo.

13. Existencia de planes de contingencia con responsabilidades y recursos previamente asignados.

14. Existencia de sistemas ágiles, justos y efectivos de solución de problemas.

15. Infraestructura de información y comunicaciones moderna, ampliamente compartida y totalmente integrada.

16. Utilización de indicadores de gestión —a todos los niveles- para la evaluación, en tiempo real, de la salud del negocio.

17. Sistemas de toma de decisiones participativos, ágiles y efectivos.

\subsection{Relaciones entre características comunes observadas}

Las características anteriormente descritas no son independientes entre sí. A continuación se sugieren grados de interrelación entre ellas, inferidos del análisis de datos y de la revisión de literatura.

4.2.1. La existencia de una estrategia corporativa pensada para la red y desagregada a nivel de cada empresa participante es el mapa de navegación que define el modelo de negocio y cómo ponerlo en práctica (David, 1990). El éxito en la ejecución de la estrategia depende de la calidad de los actores involucrados (Chiavenato, 1988), de la estructura de roles y funciones desde la cual organicen su trabajo (Mintzberg y Waters, 1985; Mintzberg y Lampel, 1999) y de la efectividad en la gestión de los recursos (Heizer y Render, 1997; Miranda-González, 2005). El ciclo estratégico se cierra con la evaluación de indicadores de resultados (Ouchi, 1979; Kaplan y Norton, 1996).

4.2.2. El modelo de negocio se hace realidad en la ejecución cotidiana de procesos. El sistema de gestión dirige la acción colectiva con criterios de coherencia y uso efectivo de los recursos para lo que se apoya en la sincronización de operaciones (Helms y Ettkin, 2000), así como del registro y evaluación de indicadores. Por otr a parte, esta acción coordinada, transcurre sobre dos plataformas tecnológicas indispensables: la red logística para el movimiento de inventarios (Ballou, 2004) y la infraestructura de información y comunicación para el procesamiento de información y la transmisión de mensajes que faciliten la toma de decisiones. 
Organizaciones en red

Factores críticos de diseño

4.2.3. La ejecución de procesos sincronizados con base en predicciones de demanda compartidas y programación conjunta es una de las cualidades que define el carácter de óptimo en la gestión de redes (Helms y Ettkin, 2000), en tanto afecta los márgenes de ganancia y operación (Ellis y McGuire, 1993). El sistema de gestión es el responsable de garantizar este estado de cosas y para hacerlo se apoya, por un lado, en sistemas efectivos de comunicación, solución de problemas, toma de decisiones, planes de contingencia e indicadores de gestión; mientras, por otro, está limitado por el diseño de la red logística que restringe las opciones de acción.

4.2.4. Los sistemas de control de calidad acordados, y que funcionan adecuadamente, son recursos críticos a procesos de mejoramiento continuo y de optimización de la red logística. Sin control no pueden garantizarse ejecuciones de calidad ni, consecuentemente, el cumplimiento de los mínimos requisitos para la competitividad. Debido a su importancia para la supervivencia del sistema, su funcionamiento adecuado es una de las responsabilidades más significativas a cargo del sistema de gestión. Al igual que con las demás actividades fundamentales, su aplicación produce registros que alimentan al sistema de indicadores.

4.2.5. La negociación de precios, basado en márgenes de ganancia, exige el conocimiento actualizado de los costos por todos los participantes.

4.2.6. El sistema de gestión se aplica a decidir sobre asuntos cotidianos y estratégicos. Entre sus responsabilidades vitales está la solución de problemas, el diseño de la red logística y la definición de perfiles de participantes. Algunos de los recursos imprescindibles para su tarea son los procesos comunicativos, la tecnología de información y comunicación, así como los indicadores de gestión.

4.2.7. La definición, comunicación y aplicación de perfiles para la selección de candidatos a participar en la red depende de las competencias y roles requeridos por el diseño organizativo.

4.2.8. El diseño de red afecta los costos de operación (Williamson, 1996) y, consecuentemente, es uno de los parámetros de entrada a los procesos de optimización. Este diseño tiene en los sistemas de comunicación a uno de sus elementos clave, por lo que las elecciones que se hagan durante el mismo afectan al sistema de toma de decisiones. 
4.2.9. El diseño de la red logística debe incluir planes de contingencia para asegurar que las operaciones integradas y altamente complejas no colapsen ante dificultades imprevistas. Por otro lado, la tecnología de información y comunicación es el mecanismo de retroalimentación por excelencia para apoyar la optimización continua del sistema.

4.2.10. Los sistemas y protocolos de comunicación, bien diseñados y efectivamente utilizados por todos los participantes, son recursos fundamentales a la toma de decisiones conjuntas y a la solución de problemas. A su vez, la tecnología de información y comunicación es la infraestructura sobre la que se apoyan los procesos comunicativos.

4.2.11. La existencia de sistemas ágiles, justos y efectivos de solución de problemas es una de las evidencias de la calidad de los sistemas de toma de decisiones de la organización.

4.2.12. La infraestructura de información y comunicación moderna, ampliamente compartida y totalmente integrada, reduce la imprecisión, lentitud y caos en los procesos de toma de decisiones en el contexto de red.

La tabla 2 ilustra las relaciones anteriormente descritas y otras de menor grado de importancia; incluye valores de 3 para relaciones consideradas fuertes (anteriormente descritas), 2 para relaciones consideradas medias, 1 para relaciones débiles y espacio en blanco para significar variables no directamente relacionadas. Una revisión detallada de esta tabla 2 nos indica que las características 3 (operación de procesos, sincronizados con base en predicciones de demanda compartidas y programación conjunta), 6 (sistema encargado de la gestión de la red, que cuente con mecanismos de representación de todos los actores) y 9 (red logística adecuadamente modelada y con mecanismos de retroalimentación que permitan la optimización dinámica) son las más conectadas (características 6 y 9) o más intensamente conectadas (características 3 y 6 ).

De las anteriores, la característica 6: Sistema encargado de la gestión de la red, que cuente con mecanismos de representación de todos los actores es la más influyente a juzgar por la intensidad y cantidad de conexiones ( 15 conexiones, 11 de las cuales son intensas). Este resultado nos indica la importancia contundente que tiene para la viabilidad, supervivencia y rentabilidad de un acuerdo productivo en red, el contar con un organismo de gestión ampliamente representativo y democrático en sus decisiones. Este ente se encarga de mirar al entramado como sistema y de garantizar que los enlaces organizacionales no solamente operen eficiente y eficazmente, si no que 
Organizaciones en red

Factores críticos de diseño

sean inclusivos y óptimos en sus configuraciones. El organismo a cargo de la conducción de los destinos de la red es su conciencia de entidad actuante en el universo de aplicación de esta estructura.

Como ejemplo de ente gestor tenemos, para el caso de los cafeteros en Colombia, a la Federación Nacional de Cafeteros. La Federación es una estructura que incluye a representantes de todos los estamentos productores; estos representantes son elegidos por votación democrática desde todos los confines del sector y sus decisiones son ampliamente consultadas y oportunamente comunicadas a los lugares y actores más remotos de la geografía. Sin la Federación, el café de Colombia no tendría el posicionamiento de marca y la calidad superior que el mundo compra. La Federación es el alma del negocio cafetero en el país y ejemplo de núcleo gestor para agrupamientos productivos en red (Villegas, 2004).

Tabla 2

Correlaciones entre condiciones ideales de una organización en red

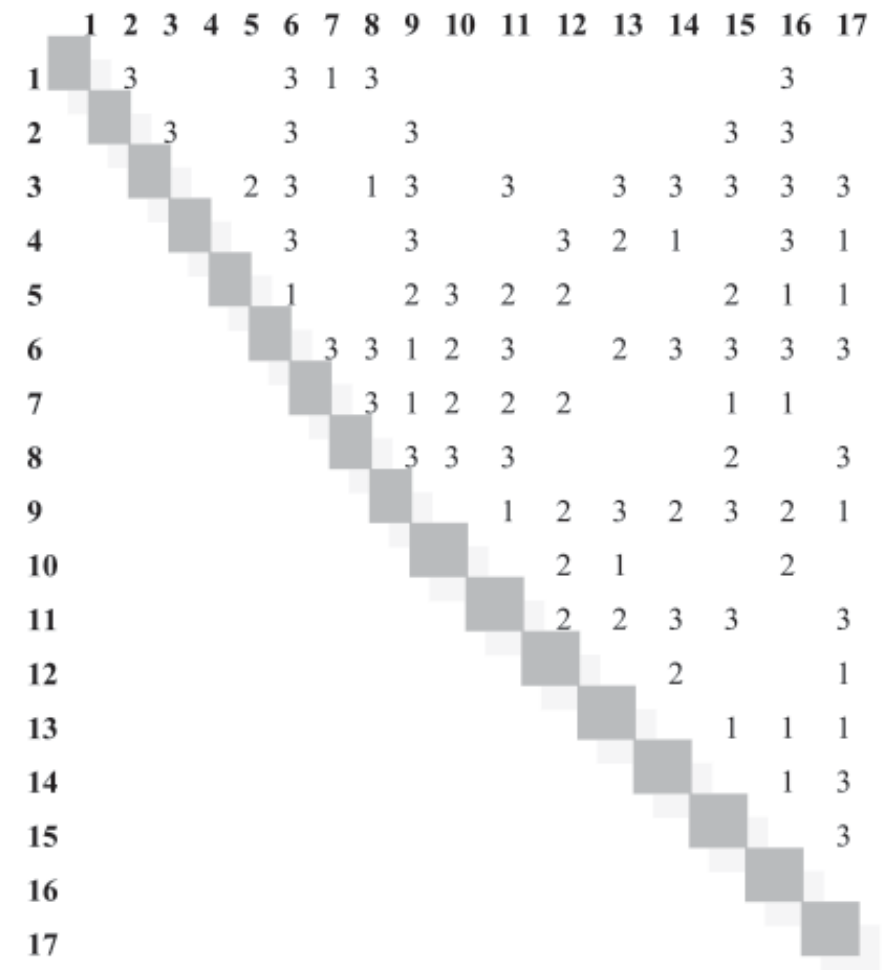

No. 225, mayo-agosto 2008: 9-38 
Seguidamente, las variables teóricas y las características observadas se relacionan, a efectos de evidenciar las variables con mayor grado de incidencia en el desarrollo de las características. Las mismas que se refinarán con base en su grado de importancia entre su grupo (dada por los análisis precedentes) para, finalmente, incluirse en un modelo de factores críticos de diseño de redes.

\subsection{Relación entre variables de diseño de redes y características observadas}

A1. La estrategia de producción responde al modelo de negocio y se deriva de la estrategia corporativa (Gaynor, 1999). Su objetivo es velar por la calidad de los procesos productivos en asuntos tan decisivos como la sincronización de operaciones. Al igual que otras actividades, se apoya en los indicadores de gestión para evaluar la efectividad en su ejecución.

A2. La programación de operaciones es una tarea decisiva al funcionamiento de la red, tanto en lo estratégico de puesta en marcha del modelo de negocio como en lo táctico de sincronización de ejecuciones conjuntas. Herramientas esenciales de apoyo al trabajo en esta actividad son los procesos de comunicación e información y sus tecnologías asociadas. Dados los altos grados de contingencia del encadenamiento productivo, los planes para atender situaciones críticas e imprevistas son absolutamente necesarios.

A3. La gestión de inventarios aplica los principios de gestión al abastecimiento de los materiales e insumos requeridos para ejecutar el modelo de negocio. Esta gestión es crucial a la optimización de la red logística, particularmente en asuntos como la sincronización de operaciones — la que no puede lograrse si se agotan los materiales requeridos.

A4. La gestión tecnológica participa de la implementación del modelo de negocio en el marco de las decisiones estratégicas vigentes. Su principal objetivo es la optimización de la operación logística. Al logro de ese propósito aplica herramientas que van desde los acuerdos para la sincronización de procesos hasta las inversiones en tecnología de información y comunicación.

A5. Las Tecnologías de Información y Comunicación (TIC) son un apoyo fundamental a la gestión moderna en tanto facilitan el registro, distribución y procesamiento de información para la toma de decisiones. Las TIC que conectan organizaciones se denominan interorganizacionales y prestan un apoyo invaluable en temas tan decisivos como la optimización de la red logística y la sincronización de procesos (Pentland y Feldman, septiembre-octubre, 2007; Kane, y Alavi, septiembre-octubre, 2007). 
Organizaciones en red

Factores críticos de diseño

B1. La planeación es una metodología de trabajo para organizar la acción a futuro. Esta forma de análisis se aplica a asuntos tales como la definición del modelo de negocio, la formulación de la estrategia y el diseño de planes de contingencia. Su complemento es la metodología de control que usa indicadores de gestión para comparar los resultados de la acción a los propósitos del plan.

B2. Las redes productivas se proyectan utilizando principios de diseño de organizaciones; sin embargo, algunos componentes del diseño deben ser repensados, tales como los perfiles de los participantes y los sistemas de toma de decisiones, debido a que en este caso los actores son organizaciones en lugar de individuos (es decir, entes independientes cuya participación es voluntaria).

B3. Los participantes se vinculan con base en los roles y perfiles definidos por el diseño organizativo. La gestión de la red será tan buena como quien la ejerza. El éxito en los procesos de negociación complejos y cotidianos, de carácter estratégico y operativo, dependen del perfil de los actores —un ejemplo de la dificultad de las decisiones cotidianas son los acuerdos para sincronizar procesos, que usualmente sacrifican óptimos locales para garantizar el bienestar total del sistema.

B4. La competitividad integra las intenciones del modelo de negocio a la ejecución de la estrategia. Para que un modelo de negocio sea exitoso, debe basarse en las competencias de la red. A su vez, es responsabilidad de la estrategia el aplicar todos los recursos para hacer a la red más competitiva. Por otro lado, competitividad e indicadores se relacionan en tanto éstos se aplican para evaluar la competitividad del sistema.

B5. Al proceso de aplicar principios de gestión para balancear la oferta a la demanda se le denomina "gestión de demanda vs. suministro". Las salidas de este balance se aplican tanto a la sincronización de procesos como a la estimación de óptimos en la operación logística.

B6. Los sistemas contables aplican la teoría contable al registro de las transacciones de negocio de tal manera que los autores de decisión puedan estimar los márgenes de ganancia y decidir sobre óptimos de negocio desde el punto de vista de la rentabilidad en las operaciones.

B7. Los recursos necesarios para la gestión se asignan a través del presupuesto, teniendo en cuenta la información registrada en el sistema contable. 
B8. La gestión de proyectos aplica la teoría de gestión a la sincronización de tiempos y secuencias de ejecución, de actividades, dedicadas al logro de un resultado, en términos de tiempo y costo predefinidos. Por otra parte, la tecnología de información y comunicación, así como los indicadores son herramientas clave al éxito de la gestión de proyectos.

B9. La actividad financiera se encarga de velar por la salud del recurso capital, sin el cual sería imposible ejecutar cualquier estrategia. La rentabilidad se garantiza con la obtención de márgenes positivos de ganancia, objetivo al cual apuntan la mayoría de las acciones, incluso la más técnica de optimización de la red.

C1. La identidad de una red se hace visible en rasgos tales como: el modelo de negocio al que se aplica, la estrategia que elige para hacerlo realidad, los perfiles con que filtra a los candidatos al sistema, los sistemas de comunicación y los estilos de toma de decisiones. Incluso, una forma fácil y rápida de reconocer la identidad organizacional es revisar el conjunto de indicadores de gestión vigentes, en tanto ellos representan los asuntos realmente prioritarios para el colectivo.

C2. Sin comunicación efectiva no hay solución posible a problemas de sistemas altamente complejos con representación de múltiples intereses y altamente interconectados. Los procesos comunicativos son el vehículo en que se transportan mensajes tanto estratégicos del tipo modelo de negocio y estrategia en uso, como operativos para los acuerdos de sincronización de actividades. Los sistemas de comunicación se plantean durante el proceso de diseño organizativo y se apoyan en tecnologías de información y comunicación.

C3. El sistema de gestión es el instrumento para la aplicación de la teoría de gestión a la conducción de la red. Su tarea primordial es la toma de decisiones en asuntos de asignación de recursos, solución de problemas, coordinación y sincronización de tareas, formulación y ejecución de planes de contingencia y optimización de la red logística, entre otros. Los indicadores de gestión apoyan el seguimiento y control a los resultados derivados de la acción.

C4. Los sistemas productivos en red son sociotécnicos. Su componente social se manifiesta en el entramado de relaciones entre actores que configuran el tejido social sobre el cual se construyen los procesos comunicativos, de toma de decisiones conjuntas, de sincronización de procesos, de negociación de márgenes de ganancia y de solución de problemas. 
Organizaciones en red

Factores críticos de diseño

C5. Sin liderazgo sería imposible gestionar una red, pues no habría suficiente poder de convocatoria para conciliar intereses diversos en bien de un objetivo común. Actividades por excelencia de los líderes son la elección de destinos estratégicos y la toma de decisiones. A su vez, procesos y sistema de comunicación y de información (especialmente indicadores de gestión) son herramientas necesarias al ejercicio de liderazgo.

C6. En estructuras productivas en red el modelo de negocio tiene que ser negociado con base en márgenes de ganancia positivos para todos los participantes. De otro modo, la red se desarticularía por deserción de actores cuya existencia no depende de la alianza y que no derivan beneficio alguno de su permanencia en ella.

C7. La confianza es el ingrediente secreto de las transacciones de negocio en la red. Si no se confía en el otro, no se comparte información de costos y sin ésta no se puede negociar con base en márgenes de ganancia; ni, consecuentemente, definir modelos de negocio colectivos. La desconfianza compromete tanto a las posibilidades de formulación estratégica como de acción inmediata sincronizada.

C8. Comprometerse es hacerse partícipe de las consecuencias de un hecho. La delegación no exime de la responsabilidad. Los gestores deben asumir las consecuencias de las ejecuciones propias y ajenas; sin embargo, todos los actores deben responsabilizarse por la calidad en su desempeño o la solución de problemas, el aseguramiento de la calidad, el mejoramiento continuo, la sincronización de tareas y la ejecución de planes de contingencia de manera exitosa no podrían garantizarse.

La tabla 3 contiene la valoración del grado de relación entre cada variable de diseño y cada característica considerada ideal para una estructura económica en red. Consistente con los análisis anteriores, un valor de 3 sugiere una relación fuerte, un valor de 2 sugiere un valor medio, un valor de 1 sugiere una relación débil y un espacio en blanco sugiere una ausencia de relación directa. 
Tabla 3

Relaciones variables de diseño vs. características de red

\begin{tabular}{|c|c|c|c|c|c|c|c|c|c|c|c|c|c|c|c|c|c|c|c|c|c|c|}
\hline & A1 & A2 & $\mathbf{A 3}$ & A4 & A5 & B1 & B2 & B3 & B4 & B5 & B6 & B7 & B8 & B9 & $\mathrm{Cl}$ & $\mathrm{C2}$ & C3 & $\mathrm{C} 4$ & C5 & C6 & C7 & $\mathrm{C} 8$ \\
\hline 1 & 3 & & 2 & 3 & & 3 & 1 & 2 & 3 & 2 & & 2 & 1 & 3 & 3 & 3 & 2 & 1 & 3 & 2 & 3 & \\
\hline 2 & 3 & 1 & 3 & 3 & 2 & 3 & & & 3 & 2 & 2 & 1 & 2 & 2 & 3 & 3 & 2 & 1 & 2 & 3 & 3 & \\
\hline 3 & 3 & 3 & 3 & 3 & 3 & 1 & 2 & 3 & 2 & 3 & 1 & 2 & 3 & & 1 & 3 & 3 & 3 & & 2 & 3 & J \\
\hline 4 & 3 & 2 & 1 & 1 & & 1 & & 2 & 2 & & & & & & 2 & 1 & 2 & 1 & & 1 & 2 & \\
\hline 5 & 1 & & & & 1 & & & & & 1 & 3 & & & 3 & & 2 & & 3 & 2 & 3 & 3 & \\
\hline 6 & 2 & 2 & 3 & 1 & 3 & 2 & 2 & 3 & 2 & 3 & 2 & 3 & 3 & 3 & 2 & 2 & 3 & 2 & 3 & 2 & 2 & 3 \\
\hline 7 & & & & & & & 3 & 3 & & & & & & & 3 & 2 & & 2 & 1 & 2 & & \\
\hline 8 & 1 & & & 1 & & 2 & 3 & 3 & 2 & & & & 2 & & & 2 & & 1 & & & & \\
\hline 9 & 1 & 1 & 3 & 3 & 3 & 1 & 1 & 1 & 2 & 3 & 3 & 2 & 1 & 3 & 2 & 2 & 3 & 1 & & 1 & 1 & \\
\hline 10 & 2 & 2 & 1 & 1 & 1 & & & & & 1 & 3 & 3 & 2 & 2 & 2 & 1 & 1 & & & 1 & 3 & \\
\hline 11 & & 3 & & 2 & 3 & & 2 & 1 & & 2 & & & & & 3 & 3 & 1 & 3 & 3 & 2 & 2 & \\
\hline 12 & 2 & 1 & 2 & 1 & & & & 1 & 2 & & & 2 & 3 & & 1 & & 2 & 1 & 2 & 1 & 1 & 3 \\
\hline 13 & & 3 & 3 & 2 & & 3 & 1 & & & 2 & & 2 & 1 & & & 2 & 3 & 1 & 1 & & 2 & 3 \\
\hline 14 & & 2 & & 2 & 2 & & & & & 1 & 1 & 1 & & & 1 & 3 & 3 & 3 & 2 & 2 & 2 & \\
\hline 15 & 1 & 3 & 2 & 3 & 3 & & 2 & 1 & 2 & 2 & 2 & 1 & 3 & 1 & 1 & 3 & 1 & 2 & 1 & & & \\
\hline 16 & 3 & 1 & 1 & 1 & 1 & 3 & & 2 & 3 & 1 & 2 & & 3 & 2 & 3 & 2 & 3 & 1 & 3 & 1 & 1 & \\
\hline 17 & & & 1 & 2 & 3 & 2 & 3 & 1 & 1 & 1 & 3 & 2 & 1 & 2 & 3 & 2 & 3 & 3 & 3 & 2 & 2 & \\
\hline
\end{tabular}

De la tabla 3 podemos inferir que las variables de diseño más decisivas al resultado son, en su orden: Proceso de Comunicación (C2), Gestión (C3) e Identidad (C1). A su vez, las características más relevantes, en su orden, son: Sistema encargado de la gestión de la red, que cuente con mecanismos de representación de todos los actores (6) y Operación de procesos, sincronizados con base en predicciones de demanda compartidas y programación conjunta (3).

Los resultados anteriores pueden interpretarse como:

- Los principios universalmente aceptados de gestión de empresas (mejores prácticas) son perfectamente aplicables a la gestión de redes, tanto a nivel estratégico (relaciones con el entorno y visión de largo plazo), como a nivel operativo (procesos de rutina dirigidos al cumplimiento de la misión del negocio). 
Organizaciones en red

Factores críticos de diseño

La viabilidad de la operación en red depende, fundamentalmente, de contar con un sistema representativo de gestión que se dedique a pensar al sistema de organizaciones como un ente con vida y lógica propias. Sin este ingrediente clave, la estructura derivaría en un caos operativo donde múltiples acciones locales darían al traste con las posibilidades de supervivencia, rentabilidad y crecimiento.

- La calidad de los sistemas de comunicación es decisiva a la gestión estratégica de la red (supervivencia, rentabilidad y crecimiento) tanto como a la gestión operativa (gestión del día a día expresada en sincronización de procesos).

La comunicación efectiva es el recurso sin el cual la asociación de empresas en redes productivas sería imposible; ello debido a que no se podría crear tejido social, persuadir a los actores de perseverar, negociar acuerdos gana-gana, coordinar operaciones, acordar estrategias ni modelos de negocios En fin, la comunicación es el ingrediente por excelencia del acuerdo entre humanos que en este caso se aplica a la operación entre empresas.

- La sincronización de procesos es la tarea más difícil y a la vez más crítica a la creación de riqueza mediante redes. Es esta armonía en la ejecución la que reduce costos, aumenta márgenes de ganancia, eleva la calidad del desempeño, reduce los tiempos de respuesta y mejora la efectividad de las decisiones. La sinergia del sistema (en este caso, creación de valor en colectivo) depende de alcanzar este ideal.

- La efectividad de la gestión de una red se ve significativamente afectada por la percepción que tienen los participantes de compartir una suerte común (identidad-espíritu de grupo). La idoneidad de las relaciones interpersonales afecta de manera decisiva a las posibilidades del trabajo conjunto.

La gráfica 1 resume los resultados del estudio: los sistemas de comunicación, los de gestión y los procesos conducentes a la construcción de identidad determinan la calidad de las relaciones entre empresas y, desde ahí, determinan la efectividad del direccionamiento estratégico y la calidad de la operación conjunta. 
Gráfica 1

Factores críticos de éxito al diseño de redes productivas

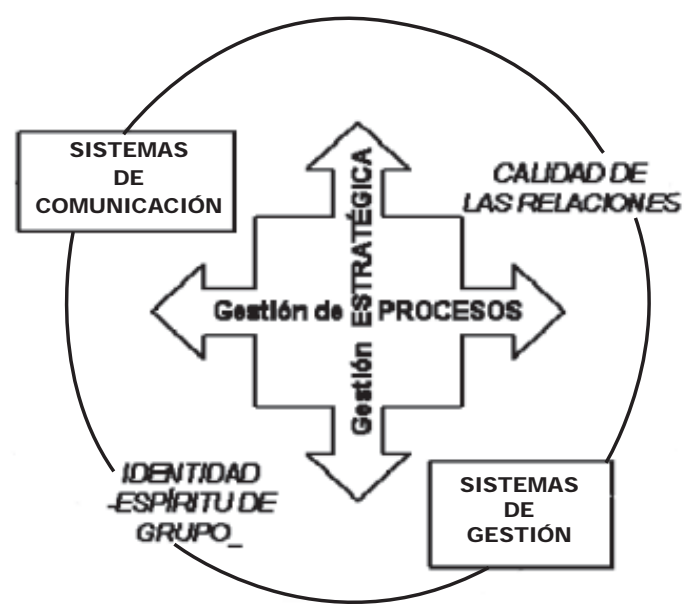

\section{Conclusiones}

La pregunta que este estudio decidió responder es ¿cómo se relacionan las características de una organización en red con las variables propias del diseño organizativo?; en particular, se deseaba:

- Identificar las características comunes a la identidad de las estructuras productivas en red.

- Reconocer las variables de diseño organizativo más influyentes cuando de diseñar ese tipo de estructuras se trata.

- Relacionar las características observadas a las variables de diseño con el fin de descubrir los factores críticos de diseño de redes.

Se descubrió que las características comunes y más relevantes en las organizaciones observadas son:

- Operación de procesos, sincronizados con base en predicciones de demanda compartidas y programación conjunta;

- Sistema encargado de la gestión de la red, que cuenta con mecanismos de representación de todos los actores y

- Red logística adecuadamente modelada y con mecanismos de retroalimentación que posibilitan la optimización dinámica. 
Organizaciones en red

Factores críticos de diseño

De entre las anteriores, el contar con un sistema encargado de la gestión de la red que cuente con mecanismos de representación de todos los actores resultó ser la características más profusa e intensamente conectada ( 15 conexiones a otras características, 11 de las cuales son intensas).

Por otra parte, la evaluación de la intensidad y cantidad de conexiones, de las variables de diseño demuestra que las más influyentes en su orden son: gestión del sistema, comunicación y gestión logística.

Finalmente, al relacionar las características de red a las variables de diseño, se encontró que los factores críticos al diseño de las redes estudiadas son, en su orden: procesos de comunicación, de gestión y de identidad, los que se aplican al desarrollar el sistema de gestión colectivo y a garantizar la sincronización de procesos conjuntamente ejecutados. Este desenlace concuerda con los hallazgos de Burke (febrero, 1997), Bus y Foreman (summer, 1993), Ernest B. (marzo, 1998), Galaskiewicz (1985), Uzzi (marzo, 1997).

Los resultados de este trabajo pueden explicarse por la importancia de las comunicaciones efectivas a la construcción de tejido social, a la coordinación de actividades y a la negociación de acuerdos; además, por la necesaria aplicación de recursos a la creación de valor, también llamada gestión y por la imprescindible necesidad que tienen los actores de sentir que hacen parte de un destino común. Todo lo anterior es decisivo al éxito de la actuación conjunta en condiciones de alta complejidad, dinamismo y precisión.

En conclusión, la existencia de una red productiva depende fundamentalmente de la efectividad en la ejecución de tres procesos sociotécnicos: comunicación, utilización de recursos y construcción de identidad, que apoyen la preservación del tejido social y desde ahí, tanto la coordinación de tareas complejas como el acierto en el direccionamiento estratégico

En tanto las redes incluidas en el estudio participan del sector agrícola colombiano, los resultados del estudio pueden no ser generalizables a redes de otros países o de otras industrias; asimismo, un tamaño de muestra de cuatro (4) no es representativo de la población mundial de redes productivas, aunque - por su tamaño y posicionamiento económico- definitivamente si lo es de esas estructuras en el país.

Otras redes deberían ser observadas con la intención de construir teoría administrativa que ayude a los gestores a obtener el mayor impacto de sus intervenciones y a los académicos a modelar estructuras asociativas novedosas. Las conclusiones de este estudio representan un paso adelante en ese sentido. 
Gladis Cecilia Villegas Arias

\section{Bibliografía}

Ballou, Ronald H. (2004), Logística, administración de la cadena de abastecimiento, 5a. ed., Prentice Hall, México, 816 pp.

BARNEY, Jay B. (1986), "Types of competition and the theory of strategy: toward and integrative framework", Academy of Management Review, 4a. ed., vol. 11, pp. 799-800.

BART, Christopher K. (1986), "Product Strategy and formal structure", Strategic Management Journal, vol. 7, núm. 4, pp. 293-312.

Barreto D., Manuel Hernán (1995), "Modalidades de outsourcing”, Revista Sistemas, 9a. ed., vol. 18.

Blanchard, K. (1996), "Turning the organizational pyramid upside down", en F. Hesselbein, M. Goldsmith y R. Beckhard (Eds.), Leader of the future, Jossey-Bass, Nueva York, pp. 81-88.

Burke, Peter J. (1997), “An identity model for network exchange”, American Sociological Review, vol. 62, febrero, pp. 1-16.

Bush, John B. y Alan Foreman (1993), "Communication in a Network Organization", Organizational Dynamics, 2a. ed., vol. 20. summer, pp. 23-36.

Chandler, Alfred D. Jr. (1998), "Corporate strategy and structure: Some current considerations", Society, vol. 35, núm. 2, pp. 347-350.

Chiavenato, Idalberto (1988), Administración de recursos humanos, McGraw Hill, México.

DAFT, R. y R. LENGEL (1984), "Information richness: a new approach to managerial behavior and organization design", en L. Cummings y B. Staw (eds.), Research in Organizational Behavior, vol. 6, pp. 191-233.

DAVID, Fred R. (1990), La gerencia estratégica, Legis, Colombia.

Egelhoof, William G. (1982), "Strategy-Structure in Multinational Corporations: An Information Processing Approach", Administrative Science Quarterly, vol. 27, núm. 3, pp. 435-458.

Ellis, Randall P. y Thomas G. McGuire (1993), "Supply side and demand side of cost sharing in Health care", Journal of Economic Perspectives, 4a. ed., vol. 7, pp. 135-151. 
Organizaciones en red

Factores críticos de diseño

Engdahl, Richard Alan; Robert J. Keating y Kenneth E. Aupperle (2000), "Strategy and Structure. Chicken or egg? (Reconsideration of Chandler's paradigm for Economic Success)", Organizational Development Journal, 4a. ed., vol. 18, pp. 21-33.

ERnest B., Alexander (1998), "Book Review: How Organizations act together: Interorganizational Coordination in Theory and Practice", Administrative Science Quarterly, vol. 43, núm. 1, marzo, pp. 217-220.

FAYOL, Henry y Frederick Winslow TAYLOR (1969), Administración industrial y general, Principios de la administración científica, El Ateneo, Buenos Aires, 210 pp.

Galaskiewicz, Joseph (1985), "Interorganizational Relations", Annual Review of Sociology, vol. 11. pp. 281-304.

GAYNoR, Gerard (1999), Manual de gestión en tecnología, tomo I, McGraw Hill, Colombia.

Grant, Robert M. (1996), "Prospering in Dynamically-Competitive Environments: Organizational Capability as Knowledge Integration”, Organization Science, 4a. ed., vol. 7, julio, pp. 375-387.

Hayes, Robert H. y Steven C. WheELwright (1984), Restoring Our Competitive Edge. Competing Through Manufacturing, John Wiley and Sons, Nueva York, 440 pp.

HARRIGAN, Kathryn Rudie (1985), "Strategies for intrafirm transfers and outside sourcing", Academy of Management Journal, 4a. ed., vol. 28, pp. 914-925.

Heizer, Jay y Barry Render (1997), Dirección de la producción, 4a. ed., Pearson Educación-Prentice Hall, Madrid.

Helms, Marilyn M. y Lawrence P. EtTKin (2000), “Time-based competitiveness: A strategic perspective", Competitiveness Review, 2a. ed., vol. 10, pp. 1-14.

Huberman, Leo (1996), Los bienes terrenales del hombre, Cometa de papel, Medellín, $315 \mathrm{pp}$.

IRENLAND, R. Duane et al. (1987), "Strategy formulation processes: Differences in perceptions of strength and weaknesses indicators and environmental uncertainty by managerial level”, Strategy Management Journal, 5a. ed., vol. 8, pp. 469-485.

Kane, Gerald C. y Maryam Alavi (2007), "Information technology and organizational learning: An investigation of exploration and exploitation processes", Organization Science, vol. 18, núm. 5, septiembre-octubre, pp. 796-812. 
KAPlan, Robert S. y David P. Norton (1996), "Using The Balance Scorecard as a Strategic Management System", Harvard Business Review, vol. 85, Iss. 7/8, enero, pp. 150-161.

Kramer, Roderick y Tom Tyler (1996), Trust in Organizations - Frontiers of theory and research, Sage Publications Inc, Londres, 429 pp.

Luhmann, Niklas (1996), Confianza, Anthropos, Barcelona.

MajchrZAK, Ann y Qianwei WANG (1996), "Breaking the functional mind-set in process organizations", Harvard Business Review, vol. 74, Issue 5, septiembre, pp. 93-93.

Meredith, Jack y Samuel J. Mantel (2007), Project Management: A Managerial Approach, 5a. ed., John Wiley y Sons, Nueva York.

MilLER, Danny (1987), "The structural and environmental correlates of business strategy”, Strategic Management Journal, vol. 8, pp. 55-76.

(1986), "Configurations of strategy and structure: Towards a synthesis", Strategic Management Journal, 3a. ed., vol. 7, pp. 233-249.

MintzBerG, Henry (1982), "La necesidad de coherencia en el diseño de la organización", Harvard DEUSTO Business Review, vol. 66, núm. 11, pp. 66-84.

y Joseph Lampel (1999), "Reflecting on the strategy process", Sloan Management Review, vol. 40, núm. 3, spring, pp. 21-30.

y James A. Waters (1985), "Of Strategies, Deliberate and Emergent”, Strategic Management Journal, vol. 6, núm. 3. pp. 257-272.

Miranda-González, Francisco Javier (2005), Manual de dirección de operaciones, Thompson, Madrid, 684 pp.

Morgan, Gareth (2006), Images of organization, 4a. ed., Sage Publications Ltda. Boston, $500 \mathrm{pp}$.

Ouchi, William G. (1979), "A conceptual framework for the design of organizational control mechanisms”, Management Science, 9a. ed., vol. 25, septiembre, pp. 833.

Pentland, Brian T. y Martha S. Feldman (2007), "Narrative networks: Patterns of technology and organization", Organization Science, vol. 18, núm. 5, septiembreoctubre, pp. 781-795.

Podolny, Joel y Karen Page (1998), "Network forms of Organization", Annual Review of Sociology, vol. 24, pp. 57-76. 
Organizaciones en red

Factores críticos de diseño

PORTER, Michael (1997), "How competitive forces shape strategy", Harvard Business Review, vol. 80, Issue 2, julio, pp. 34-50.

PRESCOTT, John E. (1986), "Environments as moderators of the relationship between strategy and performance", Academy of Management Journal, 2a. ed., vol. 29, pp. 329-346.

PridA, Bernando y Gil GuTIÉRRez (1996), "Supply Management: From Purchasing to External Factory Management", Production and Inventory Management Journal, vol. 37, pp. 38-43.

Real Academia Española (2001), Diccionario de la Lengua Española, 22a. ed., Espasa, Madrid.

Rule, John (1990), Clase obrera e industrialización, Crítica, Barcelona, 576 pp.

SipPer, Daniel y Robert Bulfin (1997), Production: Planning, Control and Integration, McGraw-Hill, Nueva York, 640 pp.

Toffler, Alvin (1993), La tercera ola, Plaza y Janes Editores, Barcelona.

(1999), El cambio del poder, Sudamericana, Barcelona.

UzzI, Brian (1997), "Social Structure and competition in Interfirm networks: The paradox of embeddeness", Administrative Science Quarterly, vol. 42, marzo, pp. $35-67$.

Venkatesan, Ravi (1992), “To make or not to make sourcing”, Harvard Business Review, vol. 70, Issue 6, noviembre, pp. 98-107.

VILLEGas, Gladis (2004), Communication media choice in developing countries (The Columbia Case), thesis (Ph.D.)-Rensselaer Polytechnic Institute, may, umI, núm. 3123017.

Walker, Gordon y David Weber (1987), "Supplier competition, uncertainty, and maker or buy decisions", Academy of Management Journal, 3a. ed., vol. 30, pp. 589-596.

Weinrich, Heinz y Harold Koontz (2004), Management: A Global Perspective, 11a. ed., McGraw-Hill Education, México, 640 pp.

WhiтE, Roderick E. (1986), "Generic Business Strategies, organizational context and performance: An empirical investigation", Strategic Management Journal, vol. 7 , pp. 217-231. 
Williamson, Oliver (1996), The mechanisms of governance, Oxford University Press. Nueva York, 429 pp. 